\title{
Preparation of some light-sensitive 2-nitrophenyl-2,3-dihydro-1H-benzodiazepines
}

\author{
Ricaurte Rodríguez, ${ }^{* a}$ Braulio Insuasty, ${ }^{b}$ Rodrigo Abonía, and Jairo Quiroga ${ }^{b}$ \\ ${ }^{a}$ Universidad Nacional de Colombia, Department of Chemistry, Grupo de Investigación en \\ Química Heterocíclica, Bogotá, A.A. 14490, Colombia \\ ${ }^{b}$ Universidad del Valle, Department of Chemistry, Grupo de Investigación de Compuestos \\ Heterocíclicos, Cali, A.A. 25360, Colombia \\ E-mail: rrodrigueza@unal.edu.co
}

\begin{abstract}
In honor of Prof. Karsten Krohn on the occasion of his $60^{\text {th }}$ birthday anniversary (received 06 Sept 04; accepted 23 Nov 04; published on the web 25 Nov 04)
\end{abstract}

\begin{abstract}
Some light-sensitive derivatives of 2,4-diaryl-7,8-dimethyl-2,3-dihydro-1H-1,5-benzodiazepines were synthesized in moderate to good yields.
\end{abstract}

Keywords: Benzodiazepines derivatives, sunlight-sensitive compounds, 1,2-diamino-3,4dimethylbenzene, $\alpha, \beta$-unsaturated carbonyl compounds

\section{Introduction}

The reaction between 1,2-diamines and $\alpha, \beta$-unsaturated carbonyl compounds is a convenient and versatile method for obtaining 1,4- and 1,5-diazepines. ${ }^{1-13}$ An alternative synthesis employs an acetophenone derivative and a 1,2-diamine (2:1 equivalents ratio) in acidic medium. ${ }^{3,4,6}$

Benzodiazepines are important compounds because of various biological activities. ${ }^{13-15}$ It is known that benzodiazepines affect the CNS, and some 2,4-diaryl-7,8-dimethyl-2,3-dihydro-1H1,5-benzodiazepines have been tested against breast cancer and have shown moderate activity. ${ }^{16}$ For these reasons we decided to synthesize nitro derivatives of the 2,4-diaryl-7,8-dimethyl-2,3dihydro-1H-1,5-benzodiazepines and in order to evaluate their biological activities that might be improved by this structural variation. In the course of the synthesis we came across an interesting feature of these derivatives. 


\section{Results and Discussion}

When the reaction was carried out under normal conditions, that is, in the presence of sunlight and under reflux, ${ }^{5,13}$ the reaction mixture was light yellow at the beginning changed to green to deep purple with time. The reaction was monitored by TLC showing a complex mixture of products that could not be separated by column chromatography.

In order to exclude any influence from light inducing a possible oxidation of 1,2-diamino3,4-dimethylbenzene and/or of o-nitrobenzaldehyde the reaction was carried out in the dark (Scheme1), and compounds 3a-f were the only products isolated in moderate to good yields.

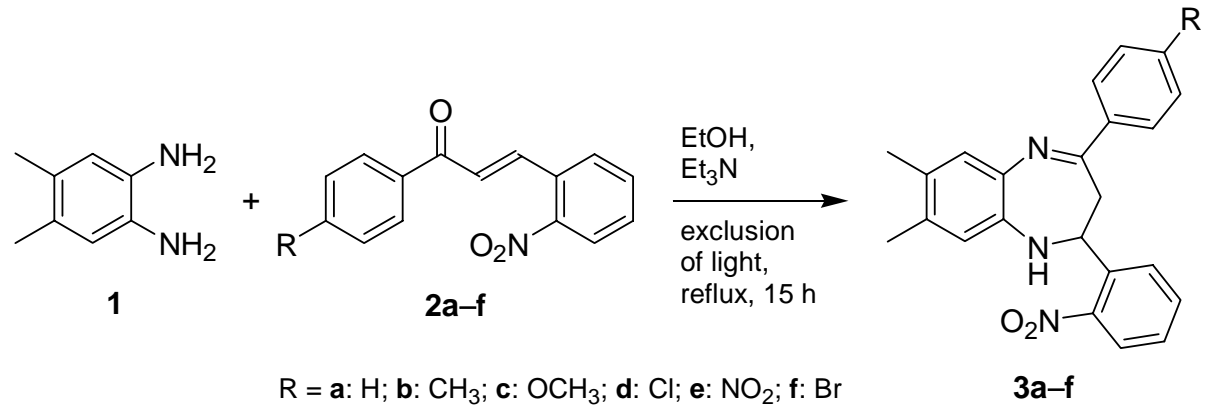

\section{Scheme 1}

The IR spectra of products 3a-f show typical bands at $3400-3415 \mathrm{~cm}^{-1}$ for the N-H, 1610$1620 \mathrm{~cm}^{-1}$ for the $\mathrm{C}=\mathrm{N}$ and $1530-1560$ and $1340-1350 \mathrm{~cm}^{-1}$ for the $\mathrm{NO}_{2}$ stretching vibrations. A superposition of other coupled $\mathrm{C}=\mathrm{C}$ vibrations of the ring system is observed in the region of $1600 \mathrm{~cm}^{-1}$. The ${ }^{1} \mathrm{H}$ NMR spectra show typical signals for these systems. The proton at $1-\mathrm{N}$ gives rise to a doublet $\left({ }^{3} J_{\text {cis }}=3.0 \pm 0.3 \mathrm{~Hz}\right.$ ) indicating the vicinal position to $2-\mathrm{H}$. In $3 \mathbf{c}-\mathbf{f}$ the geminal protons 3- $\mathrm{CH}_{2}$ exhibit two doublets of doublets with ${ }^{2} J=-13.6 \pm 0.2 \mathrm{~Hz}$ and ${ }^{3} J_{c i s}=3.7 \pm 0.3 \mathrm{~Hz}$ and ${ }^{3} J_{\text {trans }}=7.0 \pm 0.3 \mathrm{~Hz}$, and the 2-H signal is an unresolved triplet. Compounds $3 \mathbf{a}$ and $\mathbf{3 b}$ show a doublet and a triplet for $3-\mathrm{CH}_{2}$ and $2-\mathrm{H}$, respectively. The ${ }^{13} \mathrm{C}$ NMR spectra along with DEPT experiments were consistent with the structure proposed for compounds 3a-f. In order to establish unequivocally the structures microanalyses and mass spectrawere carried, out both in agreement with the proposed molecular structures. ${ }^{17}$

After the compound structures were established we carried out another experiment all products 3a-f were in dissolved in chloroform, and the solutions were exposed to sunlight. The solutions immediately changed their color indicating the light-sensitive nature of these compounds. There are reports in the literature ${ }^{18.19}$ that systems with at least one benzylic hydrogen atom in ortho position to an aromatic nitro group behave similarly.

To our knowledge there are no reports on the synthesis of benzodiazepines derivatives with sunlight-sensitive behavior; therefore, further studies are in progress in order to understand this behavior and identify the products of the photochemical reaction that takes place. 


\section{Experimental Section}

General Procedures. Melting points were determined with a digital Electrothermal, serie IA9100 melting point apparatus. The IR spectra were obtained in potassium bromide pellets on an ATI-Mattheson spectrophotometer. ${ }^{1} \mathrm{H}$ and ${ }^{13} \mathrm{C}$ NMR spectra including DEPT experiments were run on a Bruker AM 400 in DMSO- $d_{6}$ using TMS as internal standard. Mass spectra were obtained from a Varian Model MAT MS-311 spectrometer at $70 \mathrm{eV}$. Microanalyses were performed with a Perkin Elmer Model $240 \mathrm{C}$ Elemental Analyzer, and are within $\pm 0.4 \%$ of the theoretical values.

Typical procedure for 7,8-Dimethyl-2-(2-nitrophenyl)-4-phenyl-2,3-dihydro-1H-1,5benzodiazepine (3a). Under exclusion of light a solution of 1 (500 $\mathrm{mg}, 3.67 \mathrm{mmol}$ ) and 2a (929 mg, $3.67 \mathrm{mmol})$ in absolute ethanol $(15 \mathrm{~mL})$ and TEA $(3 \mathrm{~mL})$ was refluxed for $15 \mathrm{~h}$. The reaction mixture was cooled to $0{ }^{\circ} \mathrm{C}$ and left overnight. The precipitate formed was filtered off and recrystallized from ethanol affording light yellow crystals 3a (804 mg, 59\%); mp 159$161^{\circ} \mathrm{C}$. IR (KBr): $\widetilde{v} 3408(\mathrm{NH}), 1620(\mathrm{C}=\mathrm{N}), 1525$ and $1350\left(\mathrm{NO}_{2}\right) \mathrm{cm}^{-1}$. ${ }^{1} \mathrm{H} \mathrm{NMR}(400 \mathrm{MHz}$, DMSO-d $\left.)_{6}\right): \delta 2.17\left(6 \mathrm{H}, \mathrm{s}, 7-, 8-\mathrm{CH}_{3}\right), 3.16\left(2 \mathrm{H}, \mathrm{d}, J=-13.4 \mathrm{~Hz}, 3-\mathrm{CH}_{2}\right), 5.55(1 \mathrm{H}, \mathrm{dd}, J=2.7$, $6.7 \mathrm{~Hz}, 2-\mathrm{CH}), 5.71(1 \mathrm{H}, \mathrm{d}, J=2.7 \mathrm{~Hz}, \mathrm{NH}), 6.74(1 \mathrm{H}, \mathrm{s}, 9-\mathrm{H}), 6.98(1 \mathrm{H}, \mathrm{s}, 6-\mathrm{H}), 7.34(3 \mathrm{H}, \mathrm{m}$, $\left.\mathrm{H}_{\mathrm{ar}}\right), 7.44\left(1 \mathrm{H}, \mathrm{m}, \mathrm{H}_{\mathrm{ar}}\right), 7.60\left(1 \mathrm{H}, \mathrm{m}, \mathrm{H}_{\mathrm{ar}}\right), 7.73\left(1 \mathrm{H}, \mathrm{m}, \mathrm{H}_{\mathrm{ar}}\right), 7.76\left(1 \mathrm{H}, \mathrm{m}, \mathrm{H}_{\mathrm{ar}}\right), 7.89\left(1 \mathrm{H}, \mathrm{m}, \mathrm{H}_{\mathrm{ar}}\right)$.

${ }^{13} \mathrm{C}$ NMR (100 MHz, DMSO-d $\left.{ }_{6}\right): \delta 18.2,18.9\left(2 \mathrm{CH}_{3}\right), 35.4(3-\mathrm{C}), 63.5(2-\mathrm{C}), 120.7,123.1$, $123.5,127.0,128.1,128.2,128.7,130.2,131.1,133.1,134.5,134.6,137.2,138.2,139.3,147.3$, 164.3 (4-C). EI-MS m/z (\%): 371 (49) [M], 105 (100). Anal. Calcd for $\mathrm{C}_{23} \mathrm{H}_{21} \mathrm{~N}_{3} \mathrm{O}_{2}$ (371.43): C, 74.37; H, 5.70; N, 11.31. Found: C, 74.29; H, 5.74; N, 11.37.

\section{7,8-Dimethyl-4-(4-methylphenyl)-2-(2-nitrophenyl)-2,3-dihydro-1H-1,5-benzodiazepine}

(3b). Yellow crystals (608 mg, 43\%); mp 179-180 ${ }^{\circ} \mathrm{C}$. IR $(\mathrm{KBr}): \widetilde{v} 3412(\mathrm{NH}), 1615,(\mathrm{C}=\mathrm{N})$, 1528 and $1345\left(\mathrm{NO}_{2}\right) \mathrm{cm}^{-1} .{ }^{1} \mathrm{H}$ NMR $\left(400 \mathrm{MHz}, \mathrm{DMSO}-d_{6}\right): \delta 2.16\left(6 \mathrm{H}, \mathrm{s}, 7,8-\mathrm{CH}_{3}\right), 2.28(3 \mathrm{H}$, s, Aryl- $\left.\mathrm{CH}_{3}\right), 3.12\left(2 \mathrm{H}, \mathrm{t}, J=-13.6 \mathrm{~Hz}, 3-\mathrm{CH}_{2}\right), 5.53(1 \mathrm{H}, \mathrm{dd}, J=2.9,7.0 \mathrm{~Hz}, 2-\mathrm{CH}), 5.65(1 \mathrm{H}$, $\mathrm{d}, J=2.9 \mathrm{~Hz}, \mathrm{NH}), 6.73(1 \mathrm{H}, \mathrm{s}, 9-\mathrm{H}), 6.96(1 \mathrm{H}, \mathrm{s}, 6-\mathrm{H}), 7.13\left(2 \mathrm{H}, \mathrm{m}, \mathrm{H}_{\mathrm{ar}}\right), 7.44\left(1 \mathrm{H}, \mathrm{m}, \mathrm{H}_{\mathrm{ar}}\right)$, $7.60\left(1 \mathrm{H}, \mathrm{m}, \mathrm{H}_{\mathrm{ar}}\right), 7.64\left(2 \mathrm{H}, \mathrm{m}, \mathrm{H}_{\mathrm{ar}}\right), 7.78\left(1 \mathrm{H}, \mathrm{m}, \mathrm{H}_{\mathrm{ar}}\right), 7.89\left(1 \mathrm{H}, \mathrm{m}, \mathrm{H}_{\mathrm{ar}}\right) .{ }^{13} \mathrm{C} \mathrm{NMR}(100 \mathrm{MHz}$, DMSO-d $\left.)_{6}\right): \delta 18.2,18.9\left(2 \mathrm{CH}_{3}\right), 35.4(3-\mathrm{C}), 63.5$ (2-C), 120.7, 123.4, 127.1, 128.1, 128.5, $128.6,130.1,131.2,133.2,134.6,134.7,137.3,138.1,139.4,147.2,164.3$ (4-C). EI-MS m/z (\%): 385 (82) [M], 236 (100). Anal. Calcd for $\mathrm{C}_{24} \mathrm{H}_{23} \mathrm{~N}_{3} \mathrm{O}_{2}$ (385.46): C, 74.78; H, 6.01; N, 10.90. Found: C, 74.74; H, 6.05; N, 10.86 .

\section{4-(4-Methoxyphenyl)-7,8-dimethyl-2-(2-nitrophenyl)-2,3-dihydro-1H-1,5-benzodiazepine}

(3c). Yellow crystals (840 mg, 57\%); mp 187-189 ${ }^{\circ} \mathrm{C}$. IR $(\mathrm{KBr}): \widetilde{v} 3415(\mathrm{NH}), 1612,(\mathrm{C}=\mathrm{N})$, 1532 and $1347\left(\mathrm{NO}_{2}\right) \mathrm{cm}^{-1} .{ }^{1} \mathrm{H}$ NMR (400 MHz, DMSO-d $): \delta 2.17\left(6 \mathrm{H}, \mathrm{s}, 7-, 8-\mathrm{CH}_{3}\right), 3.08$, $3.14\left(2 \mathrm{H}, \mathrm{dd}, \mathrm{dd}, J=-13.7 \mathrm{~Hz}, 3-\mathrm{CH}_{2}\right), 3.76\left(3 \mathrm{H}, \mathrm{s}, \mathrm{OCH}_{3}\right), 5.51(1 \mathrm{H}, \mathrm{dd}, J=2.7,6.9 \mathrm{~Hz}, 2-\mathrm{CH})$, $5.59(1 \mathrm{H}, \mathrm{d}, J=2.7 \mathrm{~Hz}, \mathrm{NH}), 6.73(1 \mathrm{H}, \mathrm{s}, 9-\mathrm{H}), 6.94(1 \mathrm{H}, \mathrm{s}, 6-\mathrm{H}), 6.88\left(2 \mathrm{H}, \mathrm{m}, \mathrm{H}_{\mathrm{ar}}\right), 7.45(1 \mathrm{H}$, $\left.\mathrm{m}, \mathrm{H}_{\mathrm{ar}}\right), 7.61\left(1 \mathrm{H}, \mathrm{m}, \mathrm{H}_{\mathrm{ar}}\right), 7.72\left(2 \mathrm{H}, \mathrm{m}, \mathrm{H}_{\mathrm{ar}}\right), 7.79\left(1 \mathrm{H}, \mathrm{m}, \mathrm{H}_{\mathrm{ar}}\right), 7.90\left(1 \mathrm{H}, \mathrm{m}, \mathrm{H}_{\mathrm{ar}}\right) .{ }^{13} \mathrm{C} \mathrm{NMR}$ (100 MHz, DMSO-d ${ }_{6}$ ): $\delta$ 18.2, $18.9\left(2 \mathrm{CH}_{3}\right), 35.4$ (3-C), 63.5 (2-C), 120.5, 123.4, 123.5, 127.2, 
$127.9,128.2,128.8,130.3,131.1,133.2,134.5,134.6,137.3,137.9,139.4,147.2,163.9$ (4-C). EI-MS m/z (\%):401 (85) [M], 135 (100). Anal. Calcd. for $\mathrm{C}_{24} \mathrm{H}_{23} \mathrm{~N}_{3} \mathrm{O}_{3}$ (401.46): C, 71.80; $\mathrm{H}$, 5.77; N, 10.47. Found: C, 71.87; H, 5.76; N, 10.44 .

4-(4-Chlorophenyl)-7,8-dimethyl-2-(2-nitrophenyl)-2,3-dihydro-1H-1,5-benzodiazepine (3d). Yellow crystals (894 mg, 60\%); mp 167-169 ${ }^{\circ} \mathrm{C}$. IR (KBr): $\widetilde{v} 3413(\mathrm{NH}), 1619,(\mathrm{C}=\mathrm{N}), 1535$ and $1348\left(\mathrm{NO}_{2}\right) \mathrm{cm}^{-1} .{ }^{1} \mathrm{H}$ NMR $\left(400 \mathrm{MHz}, \mathrm{DMSO}-\mathrm{d}_{6}\right): \delta 2.16\left(6 \mathrm{H}, \mathrm{s}, 7-, 8-\mathrm{CH}_{3}\right), 3.13,3.20(2 \mathrm{H}$, $\left.\mathrm{dd}, \mathrm{dd}, J=-13.5 \mathrm{~Hz}, 3-\mathrm{CH}_{2}\right), 5.56(1 \mathrm{H}, \mathrm{dd}, J=3.2,7.1 \mathrm{~Hz}, 2-\mathrm{CH}), 5.77(1 \mathrm{H}, \mathrm{d}, J=3.2 \mathrm{~Hz}, \mathrm{NH})$, $6.73(1 \mathrm{H}, \mathrm{s}, 9-\mathrm{H}), 6.98(1 \mathrm{H}, \mathrm{s}, 6-\mathrm{H}), 7.39\left(2 \mathrm{H}, \mathrm{m}, \mathrm{H}_{\mathrm{ar}}\right), 7.44\left(1 \mathrm{H}, \mathrm{m}, \mathrm{H}_{\mathrm{ar}}\right), 7.60\left(1 \mathrm{H}, \mathrm{m}, \mathrm{H}_{\mathrm{ar}}\right), 7.72$ $\left(2 \mathrm{H}, \mathrm{m}, \mathrm{H}_{\mathrm{ar}}\right), 7.72\left(1 \mathrm{H}, \mathrm{m}, \mathrm{H}_{\mathrm{ar}}\right), 7.89\left(1 \mathrm{H}, \mathrm{m}, \mathrm{H}_{\mathrm{ar}}\right) .{ }^{13} \mathrm{C}$ NMR $\left(100 \mathrm{MHz}, \mathrm{DMSO}-\mathrm{d}_{6}\right): \delta 18.2,18.9$ $\left(2 \mathrm{CH}_{3}\right), 35.4$ (3-C), 63.5 (2-C), 121.1, 123.4, 123.5, 127.2, 128.1, 128.2, 128.5, 130.0, 131.2, 133.1, 134.6, 134.7, 137.2, 137.9, 139.3, 146.9, 163.1 (4-C). EI-MS m/z (\%): 405 (88) [M], 256 (100), Anal. Calcd. for $\mathrm{C}_{23} \mathrm{H}_{20} \mathrm{ClN}_{3} \mathrm{O}_{2}$ (405.88): C, 68.06; H, 4.97; N, 10.35. Found: C, 67.99; H, 4.95; N, 10.39.

7,8-Dimethyl-2-(2-nitrophenyl)-4-(4-nitrophenyl)-2,3-dihydro-1H-1,5-benzodiazepines (3e). Red crystals (963 mg, 63\%); mp 194-195 ${ }^{\circ} \mathrm{C}$. IR (KBr): $\widetilde{v} 3400(\mathrm{NH}), 1618,(\mathrm{C}=\mathrm{N}), 1560$ and $1340\left(\mathrm{NO}_{2}\right) \mathrm{cm}^{-1} .{ }^{1} \mathrm{H}$ NMR (400 MHz, DMSO-d $): \delta 2.17\left(6 \mathrm{H}, \mathrm{s}, 7-, 8-\mathrm{CH}_{3}\right), 3.18,3.35(2 \mathrm{H}, \mathrm{dd}$, $\left.\mathrm{dd}, J=-13.8 \mathrm{~Hz}, 3-\mathrm{CH}_{2}\right), 5.59(1 \mathrm{H}, \mathrm{dd}, J=3.3,7.3 \mathrm{~Hz}, 2-\mathrm{CH}), 6.01(1 \mathrm{H}, \mathrm{d}, J=3.3 \mathrm{~Hz}, \mathrm{NH})$, $6.75(1 \mathrm{H}, \mathrm{s}, 9-\mathrm{H}), 7.04(1 \mathrm{H}, \mathrm{s}, 6-\mathrm{H}), 7.43\left(1 \mathrm{H}, \mathrm{m}, \mathrm{H}_{\mathrm{ar}}\right), 7.59\left(1 \mathrm{H}, \mathrm{m}, \mathrm{H}_{\mathrm{ar}}\right), 7.64\left(1 \mathrm{H}, \mathrm{m}, \mathrm{H}_{\mathrm{ar}}\right), 7.89$ $\left(1 \mathrm{H}, \mathrm{m}, \mathrm{H}_{\mathrm{ar}}\right), 7.92\left(2 \mathrm{H}, \mathrm{m}, \mathrm{H}_{\mathrm{ar}}\right), 8.14\left(2 \mathrm{H}, \mathrm{m}, \mathrm{H}_{\mathrm{ar}}\right) .{ }^{13} \mathrm{C}$ NMR $\left(100 \mathrm{MHz}, \mathrm{DMSO}-d_{6}\right): \delta 18.2,18.9$ $\left(2 \mathrm{CH}_{3}\right), 35.4$ (3-C), 63.5 (2-C), 121.2, 123.3, 123.6, 127.1, 128.2, 128.3, 128.5, 129.9, 131.1, 133.2, 134.5, 134.6, 137.3, 138.0, 139.1, 146.8, 162.0 (4-C). EI-MS m/z (\%): 416 (75) [M], 248 (100). Anal. Calcd. for $\mathrm{C}_{23} \mathrm{H}_{20} \mathrm{~N}_{4} \mathrm{O}_{4}$ (416.43): C, 66.34; H, 4.84; N, 13.45. Found: C, 66.39; H, $4.86 ; \mathrm{N}, 13.50$.

4-(4-Bromophenyl)-7,8-dimethyl-2-(2-nitrophenyl)-2,3-dihydro-1H-1,5-benzodiazepine (3f). Yellow crystals $(1.008 \mathrm{~g}, 61 \%)$; mp 169-170 ${ }^{\circ} \mathrm{C}$. IR (KBr): $\widetilde{v} 3410(\mathrm{NH}), 1617,(\mathrm{C}=\mathrm{N}), 1545$ and $1348\left(\mathrm{NO}_{2}\right) \mathrm{cm}^{-1} .{ }^{1} \mathrm{H}$ NMR (400 MHz, DMSO-d $): \delta 2.17\left(6 \mathrm{H}, \mathrm{s}, 7-, \mathrm{CH}_{3}\right), 3.12,3.19(2 \mathrm{H}$, $\left.\mathrm{dd}, \mathrm{dd}, J=-13.5 \mathrm{~Hz}, 3-\mathrm{CH}_{2}\right), 5.55(1 \mathrm{H}, \mathrm{dd}, J=3.2,7.1 \mathrm{~Hz}, 2-\mathrm{CH}), 5.78(1 \mathrm{H}, \mathrm{d}, J=3.2 \mathrm{~Hz}, \mathrm{NH})$, $6.73(1 \mathrm{H}, \mathrm{s}, 9-\mathrm{H}), 6.98(1 \mathrm{H}, \mathrm{s}, 6-\mathrm{H}), 7.44\left(1 \mathrm{H}, \mathrm{m}, \mathrm{H}_{\mathrm{ar}}\right), 7.51\left(2 \mathrm{H}, \mathrm{m}, \mathrm{H}_{\mathrm{ar}}\right), 7.59\left(1 \mathrm{H}, \mathrm{m}, \mathrm{H}_{\mathrm{ar}}\right), 7.65$ $\left(2 \mathrm{H}, \mathrm{m}, \mathrm{H}_{\mathrm{ar}}\right), 7.72\left(1 \mathrm{H}, \mathrm{m}, \mathrm{H}_{\mathrm{ar}}\right), 7.89\left(1 \mathrm{H}, \mathrm{m}, \mathrm{H}_{\mathrm{ar}}\right) .{ }^{13} \mathrm{C} \mathrm{NMR}\left(100 \mathrm{MHz}, \mathrm{DMSO}-d_{6}\right): \delta 18.2,19.0$ (2 $\mathrm{CH}_{3}$ ), 35.4 (3-C), 63.2 (2-C), 120.8, 123.3, 123.7, 127.1, 128.2, 128.3, 128.9, 130.1, 131.0, 133.2, 134.7, 134.8, 137.4, 138.0, 139.5, 147.1, 162.0 (4-C). EI-MS m/z (\%): 450 (30) [M], 301 (100). Anal. Calcd. for $\mathrm{C}_{23} \mathrm{H}_{20} \mathrm{BrN}_{3} \mathrm{O}_{2}$ (450.33): C, 61.34; H, 4.48; N, 9.33. Found: C, 61.34; $\mathrm{H}$, $4.46 ; \mathrm{N}, 9.35$.

\section{Acknowledgements}

We are grateful to COLCIENCIAS for financial support and Professor Herbert Meier (University of Mainz) for his advices and technical collaboration. 


\section{References}

1. Nawojski, A.; Nawrocka, W. Rocz. Chem. 1977, 51, 2117; Chem. Abstr. 1978, 88, 136578.

2. Yaremenko, F. G.; Orlov, V. D.; Kolos, N. N.; Lavrushin, V. F. Khim. Geterotsikl. Soedin 1979, 848; Chem. Abstr. 1979, 91, 123717.

3. Orlov, V. D.; Quiroga, J.; Kolos, N. N. Khim. Geterotsikl. Soedin 1987, 363. Chem. Abstr. 1987, 107, 217603.

4. Orlov, V. D.; Kolos, N. N; Quiroga, J.; Kaluski, Z.; Figas, E.; Potekhin, A. Khim. Geterotsikl. Soedin 1992, 506; Chem. Abstr. 1993, 118, 38896.

5. Insuasty, B.; Abonía, R.; Quiroga, J. An. Quim. 1992, 88, 718; Chem. Abstr. 1993, 119, 49356.

6. Insuasty, B.; Abonía, R.; Quiroga, J.; Meier, H. J. Heterocycl. Chem. 1993, 30, 229.

7. Insuasty, B.; Ramos, M.; Quiroga, J.; Sánchez, A.; Nogueras, M.; Hanold, N.; Meier, H. J. Heterocyclic Chem. 1994, 31, 61.

8. Insuasty, B; Ramos, M.; Moreno, R.; Quiroga, J.; Sánchez, A.; Nogueras, M.; Hanold, N.; Meier, H. J. Heterocycl. Chem. 1995, 32, 1229.

9. Insuasty, B.; Rodríguez, R.; Quiroga, J.; Martínez, R.; Ángeles, E. J. Heterocycl. Chem. 1997, 34, 1131.

10. Insuasty, B.; Insuasty, H.; Quiroga, J.; Saitz, C.; Jullian, C. J. Heterocycl. Chem. 1999, 36, 635.

11. Insuasty, B.; Rodríguez, R.; Quiroga, J.; Abonía, R.; Saitz, C.; Jullian, C. Heterocycl. Commun. 2000, 6, 231.

12. Insuasty, B.; Rodríguez, R.; Quiroga, J.; Abonía, R.; Martínez, R.; Toscano, A.; Ángeles, E. Molecules 2001, 6, 710.

13. Roman, Gh.; Comaniță, E.; Comaniță, B. Acta Chim. Slov. 2002, 49, 575, and references cited therein.

14. Chimirri, A.; Gitto, R.; Grasso, S.; Monforte, A. M.; Romero, G.; Zappala, M. Heterocycles 1993, 36, 601, and references cited therein.

15. Sharp, J. T. In Seven-membered rings with two or more heteroatoms; Katritzky, A. R.; Rees, C. W. Eds; Comprehensive Heterocyclic Chemistry on CD ROM. Pergamon, 1997; Vol. 1, pp 595-615, and references cited therein.

16. Personal communication from National Cancer Institute, for information contact: braulio@quimica.univalle.edu.co or abonia@quimica.univalle.edu.co.

17. Quiroga, J.; Insuasty, B.; Gallo, G. Bull. Soc. Chil. Quim. 1996, 41, 415. Chem. Abstr. 1997, 126, 143853.

18. Sousa, J. A.; Weinstein, J. J. Org. Chem. 1962, 27, 3155.

19. Bluhm, A. L.; Sousa, J. A.; Weinstein, J. J. Org. Chem. 1963, 29, 636. 